\title{
Le sentiment de compétence des élèves présentant des difficultés de comportement: existe-t-il des liens avec la qualité des interactions de sa classe?
}

\section{HRONIQUE • Recherche étudiante}

\section{Introduction}

Le présent article porte sur la problématique qui guide notre projet de recherche ainsi que la méthodologie envisagée pour répondre aux objectifs de recherche.

Diverses études préconisent la participation de chaque élève à la vie scolaire et sociale de la classe ordinaire (Rousseau, Prud'homme et Vienneau, 2015). D'autres montrent que la scolarisation de tous en classe ordinaire continue de poser des défis, notamment dans le cas d'élèves présentant des difficultés comportementales (PDC [Kauffman et Landrum, 2009]). Ces élèves se caractérisent par leurs réactions comportementales ou émotionnelles différentes des normes associées à leur âge ou à leur culture et qui, par leur fréquence, leur intensité, leur durée et leur constance, ont un effet négatif sur leur rendement scolaire et sur leur fonctionnement social. Les comportements problématiques manifestés (agitation, colère, opposition, etc.) perturbent les interventions pédagogiques et peuvent modifier les apprentissages de tous les élèves de la classe (Nicholson, 2013). Des répercussions négatives (échec, exclusion, abandon) jalonnent le parcours scolaire des élèves $\mathrm{PDC}$ et se poursuivent à l'âge adulte (Kern, Hilt-Panahon et Sokol, 2009). Ces constats laissent penser que, dans le cas des élèves PDC, l'école québécoise atteint plus difficilement les finalités d'instruction, de socialisation et de qualification qu'elle s'est donnée. 


\section{Problématique}

La réussite éducative et sociale de tous les élèves est au cœur des préoccupations des chercheurs et des intervenants en éducation. Les facteurs personnels des élèves ont été identifiés comme un déterminant important de leur réussite éducative (Gouvernement du Québec, 2017). Parmi ces facteurs, le sentiment de compétence joue un rôle de premier ordre (Bandura, 2007).

Le sentiment de compétence se définit comme l'évaluation que l'élève porte sur ses capacités à atteindre un but. Il se développe entre autres au gré du succès et des échecs vécus dans les expériences (Bandura, 2007). Le milieu scolaire est donc susceptible d'influer sur le sentiment de compétence par les nombreuses expériences qui y surviennent dans la vie des élèves. Il y a lieu de penser que, l'expérience scolaire des élèves PDC étant marquée par des expériences négatives (échecs, relation difficile avec l'enseignant, rejet des pairs), leur sentiment de compétence pourrait en être affecté.

Les études démontrent en effet que les élèves qui expérimentent des difficultés répétitives sur le plan scolaire ou social sont susceptibles d'évaluer moins favorablement leurs compétences (Bussing, Zima et Perwien, 2000). Le sentiment de compétence peut aussi être affecté négativement lorsque les élèves vivent des relations conflictuelles avec l'enseignant (Baek et Yoo, 2017). À l'inverse, lorsque l'enseignant utilise des rétroactions positives et constructives envers l'élève, il tend à accroitre son sentiment de compétence (Ryan et Deci, 2000). Dans ces circonstances, les interactions de classe apparaissent comme une variable à considérer dans l'étude du sentiment de compétence des élèves PDC. Pour notre recherche, c'est le modèle de Pianta, La Paro et Hamre (2008), définissant la qualité des interactions de classe selon trois domaines (soutien émotionnel, organisation de la classe et soutien à l'apprentissage), qui a été retenu.

Que ce soit à partir de ce modèle ou d'un autre, le portrait de la qualité des interactions est peu étayé au Québec. Les études sont limitées, autant au préscolaire (Duval, Bouchard, Hamel et Pagé, 2016) qu'au secondaire (Poulin et Lessard, 2016), et semblent absentes du primaire. De surcroit, la plupart d'entre elles ont évalué la qualité des interactions à partir d'observations en dépit du fait que ce sont les perceptions des élèves qui sont déterminantes pour comprendre leur comportement (Bandura, 2007).

Or, aucun instrument n’est disponible en français pour la mesure de la qualité des interactions de classe à partir de la perception de l'élève du primaire. Un des objectifs de cette étude vise à pallier ce manque en développant un instrument de mesure quantitatif francophone. Ce faisant, elle pourra examiner les liens entre le sentiment de compétence et la qualité des interactions telle que perçue. Enfin, elle permettra d'évaluer si des différences s'observent entre les valeurs rapportées par les élèves PDC et celles données par les élèves tout venant concernant leur sentiment de compétence et leurs perceptions de la qualité des interactions.

\section{Méthodologie}

Cette recherche prévoit un devis exploratoire descriptif quantitatif. D’abord, à la suite d'une recension d'instruments existants sur les interactions de classe, un questionnaire adapté à l'élève sera rédigé. Cette version préliminaire sera soumise à un comité d'experts universitaires pour évaluer la pertinence scientifique. Elle sera soumise à des enseignants et à des élèves du primaire pour vérifier l'adéquation 


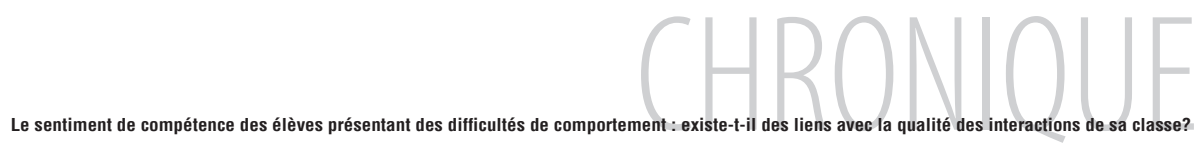

du vocabulaire et sera révisée au besoin. Par la suite, des élèves de la $4^{\mathrm{e}}, 5^{\mathrm{e}}$ et $6^{\mathrm{e}}$ année du primaire seront invités à remplir la version finale comportant des questions sur la qualité des interactions de classe et d'autres sur leur sentiment de compétence. Des analyses statistiques descriptives, de variance et corrélationnelles permettront d'atteindre les objectifs.

\section{Conclusion}

Si le sentiment de compétence et la qualité des interactions de classe ont chacun reçu une attention soutenue dans la littérature scientifique, peu d'études ont examiné les liens entre ces notions chez les élèves PDC. Les connaissances issues de ce projet de recherche permettront de mieux comprendre le vécu scolaire des élèves PDC en évaluant leur sentiment de compétence et en décrivant leurs perceptions de la qualité des interactions de classe. Ultimement, ces connaissances pourront aider les enseignants et les intervenants scolaires à cibler des pistes de soutien novatrices et pertinentes.

\section{Références}

Baek, S. et Yoo, H. (2017). Ecological factors influencing emotional/behavioral problems and self-concept in adolescents from low-income families in South Korea. Issues in Mental Health Nursing, 38(9), 733-741. http://dx.doi.org/10.1080/01612840.2017.1325949

Bandura, A. (2007). Auto-efficacité. Le sentiment d'efficacité personnelle. Paris : De Boeck.

Bussing, R., Zima, B. T. et Perwien, A. R. (2000). Self-esteem in special education children with ADHD: Relationship to disorder characteristics and medication use. Journal of the American Academy of Child E Adolescent Psychiatry, 39(10), 1260-1269. http://dx.doi.org/10.1097/00004583-200010000-00013

Duval, S., Bouchard, C., Hamel, C. et Pagé, P. (2016). La qualité des interactions observées en classe et les pratiques déclarées par les enseignantes à l'éducation préscolaire. Revue canadienne de l'éducation, 39(3), 1-27. Repéré à http://journals.sfu.ca/cje/index.php/cje-rce/article/view/2286

Gouvernement du Québec. (2017). Politique de la réussite éducative. Le plaisir d’apprendre, la chance de réussir. Repéré à http://www.education.gouv.qc.ca/fileadmin/site web/documents/PSG/politiques orientations/politique reussite educative 10juillet F 1.pdf

Kauffman, J. M. et Landrum, T. J. (2009). Politics, civil rights, and disproportional identification of students with emotional and behavioral disorders. Exceptionality, 17(4), 177-188. http://dx.doi.org/10.1080/09362830903231903

Kern, L., Hilt-Panahon, A. et Sokol, N. G. (2009). Further examining the triangle tip: Improving support for students with emotional and behavioral needs. Psychology in the Schools, 46(1), 18-32. http://dx.doi.org/10.1002/pits.20351

Nicholson, T. (2013). Academic achievement and behavior. Dans P. Garner, K. James et E. Julian (dir), The SAGE handbook of emotional and behavioral difficulties (p. 177-188). Londres : SAGE. http://dx.doi.org/10.4135/9781446247525.n13

Pianta, R. C., La Paro, K. M. et Hamre, B. K. (2008). Classroom assessment scoring system [CLASS] manual: Pre-K. Baltimore, $\mathrm{MD}$ : Brookes Publishing.

Poulin, C. et Lessard, A. (2016). Gérer la classe c'est plus que gérer les comportements : comment atteindre l'équilibre? La foucade, 17(1), 14-16. Repéré à https://crires.ulaval.ca/work/4518

Rousseau, N., Prud'homme, L. et Vienneau, R. (2015). C'est mon école àà moi aussi... Les caractéristiques essentielles de l'école inclusive. Dans N. Rousseau (dir.), La pédagogie de l'inclusion scolaire : un déf ambitieux, mais combien stimulant (3éd., p. 5-48). Québec, QC : Presses de l'Université du Québec. 
Ryan, R. M. et Deci, E. L. (2000). Self-determination theory and the facilitation of intrinsic motivation, social development, and well-being. American Psychologist, 55(1), 68-78. http://dx.doi.org/10.1037//0003-066x.55.1.68

\section{Pour citer cet article}

Beaudoin, M. (2019). Le sentiment de compétence des élèves présentant des difficultés de comportement : existe-t-il des liens avec la qualité des interactions de sa classe? Formation et profession. 27(3), 117-120.

http://dx.doi.org/10.18162/fp.2019.a188 